\title{
MicroRNA miR-466 inhibits Lymphangiogenesis by targeting prospero-related homeobox 1 in the alkali burn corneal injury model
}

\author{
Minkoo Seo ${ }^{1}$, Jun-Sub Choi ${ }^{2}$, Chang Rae Rho ${ }^{2,3}$, Choun-Ki Joo ${ }^{2,4}$ and Suk Kyeong Lee ${ }^{\text {* }^{*}}$
}

\begin{abstract}
Background: Lymphangiogenesis is one of the major causes of corneal graft rejection. Among the lymphangiogenic factors, vascular endothelial growth factor (VEGF)-C and -D are considered to be the most potent. Both bind to VEGF receptor 3 (VEGFR3) to activate Prospero homeobox 1 (Prox1), a transcription factor essential for the development and maintenance of lymphatic vasculature. MicroRNAs (miRNAs) bind to the 3 ' untranslated regions (3' UTRs) of target genes in a sequence-specific manner and suppress gene expression. In the current study, we searched for miRNAs that target the pro-lymphangiogenic factor Prox1.
\end{abstract}

Results: Among the miRNAs predicted by the bioinformatic analysis to seed match with the 3' UTR of Prox-1, we chose 3 (miR-466, miR-4305, and miR-4795-5p) for further investigation. Both the miR-466 and miR-4305 mimics, but not the miR-4795-5p mimic, significantly reduced the luciferase activity of the Prox-1 3' UTR reporter vector. In primary lymphatic endothelial cells (HDLEC), miR-466 mimic transfection suppressed Prox1 mRNA and protein expression, while miR-4305 mimic transfection did not. Experiments using mutated reporter constructs of the two possible seed match sites on the 3' UTR of Prox 1 suggested that the target site 2 directly bound miR-466. HDLEC transfected with the miR-466 mimic suppressed tube formation as compared to the scrambled control. Furthermore, HDLEC transfected with a miR-466 inhibitor showed enhanced tube formation as compared to control inhibitor transfected cells, and this inhibitory effect was counteracted by Prox 1 siRNA. The miR-466 mimic reduced angiogenesis and lymphangiogenesis resulting in clearer corneas in an cornea injury rat model compared to the scrambled control.

Conclusions: Our data suggest that miR-446 may have a protective effect on transplanted corneas by suppressing Prox 1 expression at the post-transcriptional level. The results of the current study may provide insights into the mechanisms of lymphangiogenesis resulting from corneal graft rejection and alkali-burn injuries, as well as into the development of new treatments for lymphangiogenic eye diseases.

Keywords: MicroRNA, Prox1, miR-466, miR-181, Tube Formation, Lymphangiogenesis, Cornea transplantation, Alkali burn

\section{Background}

Approximately $10 \%-50 \%$ of cornea transplantation recipients experience graft rejection within one year [1]. Corneal graft rejection takes place when the immune cells of the host recognize the donor tissues as antigens and attack them. The normal cornea maintains avascularity by balancing positive and negative angiogenesis-regulating molecules such as vascular endothelial growth factor (VEGF)

\footnotetext{
* Correspondence: sukklee@catholic.ac.kr

'Department of Medical Lifescience, College of Medicine, The Catholic University of Korea, Seoul, Korea

Full list of author information is available at the end of the article
}

and angiostatin, respectively. Stimulation of cornea caused by corneal transplantation promotes the production of VEGF, disrupts the balance, and results in capillary endothelial cell proliferation and neovascularization [2,3]. Corneal lymphangiogenesis is also induced after corneal transplantation [4]. While the blood vessels provide a route of entry for $\mathrm{CD}^{+}$alloreactive $\mathrm{T}$ lymphocytes and memory $\mathrm{T}$ lymphocytes [5], newly formed corneal lymphatic vessels enable effective access of antigen presenting cells and antigenic materials to lymph nodes where accelerated sensitization to graft antigens occurs [6]. 
Angiogenesis, defined as the sprouting of new blood vessels from existing blood vessels, is promoted by proangiogenic factors, such as VEGF, angiopoietins, and integrins [7]. The potent angiogenic inducer VEGF-A can bind to both VEGF receptor 1 (VEGFR1) and VEGFR2. However, the signals responsible for inducing proliferation and migration of vascular endothelial cells are mainly transduced via VEGFR2 [8].

Lymphangiogenesis is known to be closely associated with inflammation, wound healing, corneal graft rejection, and tumors. The cell survival, proliferation, and migration of epithelial cells are important process in lymphangiogenesis, which depends on VEGF-C and -D signalling pathways through VEGFR-2 and VEGFR-3 $[9,10]$. Especially, VEGF-C and $-D$ bind with VEGFR-3 and activate Prospero homeobox 1 (Prox1) [11]. Prox1 is homolog of the drosophila homeobox protein prospero [12]. Prox1 is a transcription factor essential for the embryonic development of vertebrates, and the development and maintenance of lymphatic vasculature in adulthood [13-15].

Following transplantation of corneas from C57BL/6 mice into BALB/C mice, the graft survival rates were compared between two experimental groups [16]. In one group, VEGF-TrapR1R2 was used to inhibit both lymphangiogenesis and angiogenesis, and in the other, VEGFR3 Ab mF4-31C was used to inhibit lymphangiogenesis only. Results showed that the survival rates of the corneal grafts were comparable in the two groups, indicating that lymphangiogenesis but not angiogenesis was an important determinant for graft survival rates.

Inflammation-induced lymphangiogenesis has been reported to be attributable to the increased expression of Prox1 stimulated by inflammatory responses [17]. In particular, Prox1 promotes the expression of the VEGF-C receptor, VEGFR3 [18]. In Prox $1^{+/-}$mice, milky chyle leaked from the mesenteric lymphatic vessels, and abnormal lymphatic ducts were formed [19]. Furthermore, embryos of Prox1-knockout mice showed a loss of lymphangiogenesis without disrupted hemangiogenesis from the cardinal vein [13]. Therefore, inhibiting Prox1 function or reducing Prox1 expression may be effective strategies for inhibiting corneal lymphangiogenesis.

MicroRNAs (miRNAs) are highly conserved small noncoding RNAs (19-25 nucleotides) that can modulate gene expression. Primary miRNA transcripts are processed consecutively to produce mature miRNAs by the two RNase III endonucleases, Drosha and Dicer. Mature miRNAs function as negative gene regulators through complementary sequence pairing with the 3 ' untranslated regions (3' UTRs) of target genes [20].

Kazenwadel et al. [21] reported that the over-expression of miR-181a in mouse lymphatic endothelial cells directly targeted the 3' UTR of Prox1, and the expression of miR181a was lower in vascular endothelial cells than in lymphatic endothelial cells. Furthermore, the expression of miR-181a was inversely related to the expression of Prox1 [21]. Other investigators found that miR-31 targets the 3' UTR of Prox1 to suppress its expression in human lymphatic endothelial cells, and that over-expressed miR31 led to defective lymphangiogenesis in Xenopus and zebrafish embryos [22]. However, there may be other unknown miRNAs capable of down-regulating Prox1 expression as well.

In the current study, miR-466, miR-4305, and miR4795-5p were chosen as new miRNA candidates that could target the 3' UTR of Prox1 based on the results of a bioinformatics analysis. The ability of these miRNAs to suppress the expression of Prox 1 in vitro was then investigated. The in vivo inhibitory effects of these miRNAs on lymphangiogenesis were also assessed using an experimental alkali corneal burn animal model.

\section{Methods \\ Cells}

Human dermal lymphatic endothelial cells (HDLEC) were purchased from PromoCell (Heidelberg, Germany) and cultured in MV2 media (PromoCell). HEK293T were cultured in DMEM (Gibco BRL, Grand Island, NY, USA) supplemented with $10 \%$ fetal bovine serum and antibiotics $(100 \mathrm{U} / \mathrm{mL}$ penicillin and $100 \mu \mathrm{g} / \mathrm{mL}$ streptomycin; Gibco BRL). Both cells were incubated at $37^{\circ} \mathrm{C}$ and supplemented with $5 \% \mathrm{CO}_{2}$.

\section{miRNA mimics, siRNA, and miRNA inhibitor}

The miRNA mimics, siRNA, and scrambled miRNA used as a negative control were purchased from Genolution Pharmaceuticals (Seoul, South Korea). The sequences are as follows: scrambled control sense, $5^{\prime}$-UUUUAACUCA GUAUUUUUA-3' and antisense, 5' -UAAAAAUACUGA GUUAAAA-3'; Prox1 siRNA sense, 5' - GAGUUGACA UUGGAGUGAA-3' and antisense, 5' - UUCACUCC A AUGUCAACUC-3'. The LNA $^{\mathrm{m}}$ microRNA Power Inhibitor for hsa-miR-466 and the negative control inhibitor (NC inhibitor) were purchased from Exiqon (Vedbaek, Denmark). The sequences are as follows: inhibitor for hsa-miR-466, 5'-GTGTTGCGTGTATGTGTA-3'; NC inhibitor, 5'-GTGTAACACGTCTATACGCCCA-3'.

\section{Plasmid construction and site-directed mutagenesis}

The full length 3' UTR of Prox1 was amplified from the genomic DNA of HEK293T cells and cloned between the Renilla luciferase coding sequence and the poly(A) site of the psiCHECK-2 plasmid (Promega, Madison, WI, USA) using XhoI/NotI sites to produce psiC-Prox1. The primers used for the amplification of 3' UTR of Prox1 were as follows: 5'-TCGACTCGAGTGCCTACAAGAGCTGCTTC A-3' and 5'-GGCCGCGGCCGCATTTGGCCTTTTGG GGTACT-3'. Mutations were introduced into the putative 
seed match sequences of psiC-Prox1 using an EZchange site-directed mutagenesis kit (Enzynomics, Daejeon, South Korea). The sequences are as follows: psiCProx1-m1, 5'-AATGACTTATATATGAAATCAAAATCTA GACACAT-3' and 5'-GGGAGGCATGGATATGTTATG-3'; psiC-Prox1-m2, 5'-TTATGACTCGCCAACATTCTTTTTC3 ' and 5'-GTCTCTATTAGCAATGAAGGGAATTTGT-3'.

\section{Luciferase reporter assay}

To test whether the miRNAs directly target the 3' UTR of Prox1, luciferase reporter assay was carried out. For this, HEK293T cells were seeded in a 96-well plate $(5 \times$ $10^{3}$ cells/well). After $24 \mathrm{~h}$, cells were co-transfected with $20 \mathrm{ng}$ psiC-Prox1 or its mutants (psiC-Prox1-m1, psiCProx1-m2, and psiC-Prox1-m1m2), and $10 \mathrm{nM}$ each of the miRNA mimics. Luciferase activities were measured $48 \mathrm{~h}$ post-transfection using the Dual-Glo ${ }^{\mathrm{Tm}}$ luciferase reporter assay system (Promega). Renilla luciferase activity was normalized using firefly luciferase activity for each sample.

\section{Transfection of HDLEC}

Cells were seeded $24 \mathrm{~h}$ prior to transfection in 60 - or 100-mm-diameter dishes containing $10 \mathrm{~mL}$ culture medium. Transfection was performed with $20 \mathrm{nM}$ each of miRNA mimic, siRNA, and/or miRNA inhibitor using Lipofectamine $^{\text {Tx }} 2000$ (Invitrogen, Carlsbad, CA, USA) according to the manufacturer's protocol. Cells were harvested for RNA and protein extraction $48 \mathrm{~h}$ after transfection.

\section{Quantitative reverse transcription-polymerase chain reac- tion (qRT-PCR)}

HDLEC were harvested and total RNA was extracted using the RNAzol ${ }^{\mathrm{Tw}} \mathrm{B}$ reagent (Tel-Test, Friendswood, TX, USA) according to the manufacturer's instruction. cDNA was synthesized using $1 \mu \mathrm{g}$ total RNA, oligo(dT) (Macrogen, Seoul, South Korea), and M-MLV reverse transcriptase (Invitrogen). Real-time PCR for Prox1 was carried out using a SYBR green qPCR kit (Takara, Tokyo, Japan) with an Mx3000P ${ }^{\text {in }}$ Real-Time PCR System (Stratagene, La Jolla, CA, USA). The sequences of the primers were as follows: Prox1; 5'-ATCCCAGCTCCAATATGCTG-3' and 5'-GTACTGGTGACCCCATCGTT3', glyceraldehyde phosphate dehydrogenase (GAPDH); 5' - ATGGGGAAGGTGAAGGTCG-3' and 5'- GGGG TCATTGATGGCAACAATA-3'. The PCR conditions were $95^{\circ} \mathrm{C}$ for $10 \mathrm{~min}$, followed by 40 cycles at $95^{\circ} \mathrm{C}$ for $20 \mathrm{~s}, 60^{\circ} \mathrm{C}$ for $30 \mathrm{~s}$, and $72^{\circ} \mathrm{C}$ for $30 \mathrm{~s}$. To confirm specific amplification of the PCR product, dissociation curves were checked routinely. For this, the PCR products were incubated at $95^{\circ} \mathrm{C}$ for $10 \mathrm{~s}$ and ramped up from $55^{\circ} \mathrm{C}$ to $95^{\circ} \mathrm{C}$ with a heating rate of $0.1^{\circ} \mathrm{C} / \mathrm{s}$, and fluorescence was measured continuously. Relative gene expression was calculated according to the comparative $\mathrm{Ct}$ method using GAPDH as an internal standard.

\section{Western blot analysis}

To detect the Prox1 protein, cell lysate in RIPA buffer $(50 \mu \mathrm{g})$ was mixed with NuPAGE LDS sample buffer $(4 \times)$ and heated at $70^{\circ} \mathrm{C}$ for $10 \mathrm{~min}$. The samples were electrophoretically separated on 8\% SDS-PAGE gel, and then transferred to a nitrocellulose membrane (Invitrogen). The membrane was incubated overnight at $4^{\circ} \mathrm{C}$ with mouse monoclonal antibody against Prox1 (1:500, Abnova, Taipei City, Taiwan). After washing, the blots were incubated for $2 \mathrm{~h}$ at room temperature with horseradish peroxidase-conjugated anti-mouse secondary antibody (1:5000, Santa Cruz Biotechnology, Dallas, TX, USA). Protein bands were visualized using an enhanced chemiluminescence detection system (Amersham Biosciences). $\beta$-Actin antibody (Cell Signaling Technology, Danvers, MA, USA) was used to confirm comparable loading. The density of each protein band was read and quantified using Fujifilm Multi Gauge software (version 3.0).

\section{Tube formation assay}

Endothelial cells plated on a reconstituted basement membrane matrix have been known to rapidly attach, align, and form capillary-like tubules [23]. As this endothelial cell specific process is rapid and quantifiable, tube formation assay has been used to study angiogenic and anti-angiogenic factors, to investigate mechanisms of angiogenesis, and to define endothelial cell populations [23]. To assess the effect of miRNAs on lymphangiogenesis of HDLEC, tube formation experiments were performed using MILLIPORE ${ }^{\bullet}$ In Vitro Angiogenesis Assay Kit (MILLIPORE, Billerica, MA, USA) according to the manufacturer's protocol. Ninety six-well plates were coated with cold liquid ECMatrix $(70 \mu \mathrm{l} /$ well $)$ and incubated at $37^{\circ} \mathrm{C}$ in a humidified $5 \% \mathrm{CO}_{2}$ incubator for $1 \mathrm{~h}$ to promote solidification. miRNA-transfected cells $\left(7 \times 10^{3}\right.$ cells/well $)$ were seeded into 96-well plates pre-coated with polymerized ECMatrix and incubated with conditioned media at $37^{\circ} \mathrm{C}$ for $4-6 \mathrm{~h}$. Formation of tube-like structures was observed under a phase-contrast microscope and quantified by counting the number of tubes formed in 3 randomly chosen fields using ImageJ software.

\section{Experimental corneal alkali burn animal model}

Male Sprague-Dawley rats (body weight, approximately 250-300 g) were used in this study. All of the animals were treated in accordance with the guidelines of the Association for Research in Vision and Ophthalmology (ARVO) Statement for the Use of Animals in Ophthalmic and Vision Research, and the study protocol was approved by the Committee for Animal Research, Catholic 
University of Medicine. The rats were deeply anesthetized via intraperitoneal injection of $50 \mathrm{mg} / \mathrm{kg}$ tiletamine plus zolazepam (Zoletil; Virbac, Carros, France) and $15 \mathrm{mg} / \mathrm{kg}$ xylazine hydrochloride (Rompun; Bayer, Leuverkeusen, Germany). Alkali injuries to the eyes were induced via $10 \mathrm{~s}$ exposure of the central cornea to a 4-mm-diameter disk of filter paper soaked in $1 \mathrm{~N} \mathrm{NaOH}$, followed by rinsing with sterile saline $(10 \mathrm{~mL})$. To avoid any corneal infection, one drop of antibiotic (0.5\% levofloxacin; Cravit; Santen, Osaka, Japan) was instilled onto the ocular surface immediately after the alkali burn injury. The animals were then randomly allocated to three treatment groups: scrambled control, miR-181a, and miR-466. Each group $(\mathrm{n}=10)$ was treated with a single subconjunctival injection with $20 \mu \mathrm{l}$ of $20 \mathrm{nM}$ miRNA mimic immediately after the alkali burn injury.

\section{Immunostaining}

Formalin-fixed corneas from each group of animal were embedded in paraffin and $4 \mu \mathrm{m}$ sections were prepared for examination. To access lymphangiogenesis, corneal sections were stained with and anti-mouse lymphatic vessel endothelial hyaluronan receptor (LYVE)-1 antibody (1:500; Abcam, Eugene, OR, USA) for $16 \mathrm{~h}$ at $4^{\circ} \mathrm{C}$. After three washes with PBS for $15 \mathrm{~min}$, the sections were then stained with a Texas Red-conjugated secondary antibody (Abcam). To detect F-actin, corneal sections were incubated with rhodamine-conjugated phalloidin (dilution 1:500, Abcam) for $1 \mathrm{~h}$ and washed three times with PBS. The stained sections were incubated with hoechst solution to stain nucleus before examined by fluorescence microscopy at $100 \times$ magnification.

\section{Results}

\section{Screening of miRNAs that can target Prox 1}

In order to screen miRNAs which may target Prox1, we used publicly available TargetScan program (http://www. targetscan.org) and found 17 human microRNAs that showed a good seed match with the 3' UTR of human Prox1 mRNA (Additional file 1: Table S1). Among the 17 miRNAs, we selected miR-4305 and miR-4795-5p for further study, as they both showed 8mer seed matches with the 3' UTR of human Prox1(Figure 1A). This was considered to be important due to similarity with miR181a, which was previously shown to target Prox1. Additionally, miR-466 was also selected, as the 3' UTR of Prox1 contained two putative binding sites for this miRNA (7mer-m8 and 7mer-1A sites), unlike other miRNAs. Although miR-4262 showed an 8mer seed match with the 3' UTR of Prox1, it was excluded from further study because the 8 mer seed sequence of miR-4262 was identical to that of miR-181a. As the target sequences of miRNAs are frequently conserved in many species, we analyzed conservation of the seed match sequences in the 3' UTR of Prox1. The 7mer-1A site complementary to the target site 2 of miR-466 and the 8 mer site complementary to the seed region of miR- 4305 were well conserved among species (Figure 1B). However, the 7 mer-m8 site complementary to the target site 1 of miR466 and the 8 mer site complementary to the seed region of miR-4795-5p were not well conserved. Subsequently, a luciferase reporter assay was conducted to assess whether miR-466, miR-4305, and miR-4795-5p directly targeted the 3' UTR of Prox1. First, HEK293T cells were co-transfected with psiC-Prox1 and each miRNA mimic. miR-181a, which is known to target Prox1, was included as a positive control, while a scrambled miRNA was used as a negative control. As expected, miR-181a significantly reduced the luciferase activity of psiC-Prox 1 as compared to the scrambled control. The miR-466 and miR-4305 mimics, but not the miR-4795-5p mimic, significantly reduced the luciferase activity of the reporter vector as compared to that of the scrambled control (Figure 1C).

\section{Effect of miRNA mimics on the expression of Prox1 mRNA and protein}

To test whether miR-466 and miR-4305 have the ability to modulate Prox1 expression, HDLEC were harvested $48 \mathrm{~h}$ after miRNA mimic transfection. qRT-PCR revealed that the Prox1 mRNA level was reduced by approximately $50 \%$ following transfection with the miR-181a mimic. Similarly, the Prox1 mRNA level was decreased by about $50 \%$ following transfection with the miR- 466 mimic as compared with the scrambled control (Figure 2A). miR-4305 transfection did not affect Prox1 mRNA level significantly. Western blot analysis also showed that the level of Prox1 protein was reduced by transfection with the miR-181a and miR-466 mimics as compared to levels observed following transfection with the scrambled control (Figure 2B). However, the level of Prox1 protein was not significantly affected by miR- 4305 .

\section{Dose-dependent effect of the miR-466 mimic}

As non-specific effects can obscure the results of miRNA mimic transfection experiments, we carried out a luciferase assay using increasing doses of the miR-466 mimic. To accomplish this, HEK293T cells were cotransfected with increasing concentrations of the miR-466 mimic and psiC-Prox1 reporter plasmid. Transfection with $5 \mathrm{nM}$ miR-466 slightly reduced the luciferase activity, however the reduction was not statistically significant as compared to the scrambled control transfection (Figure 3). Transfecting the cells with $10 \mathrm{nM}$ or higher concentrations of the miR-466 mimic caused a dose-dependent reduction in the luciferase activity of psiC-Prox1 (Figure 3). To minimize any possible non- 


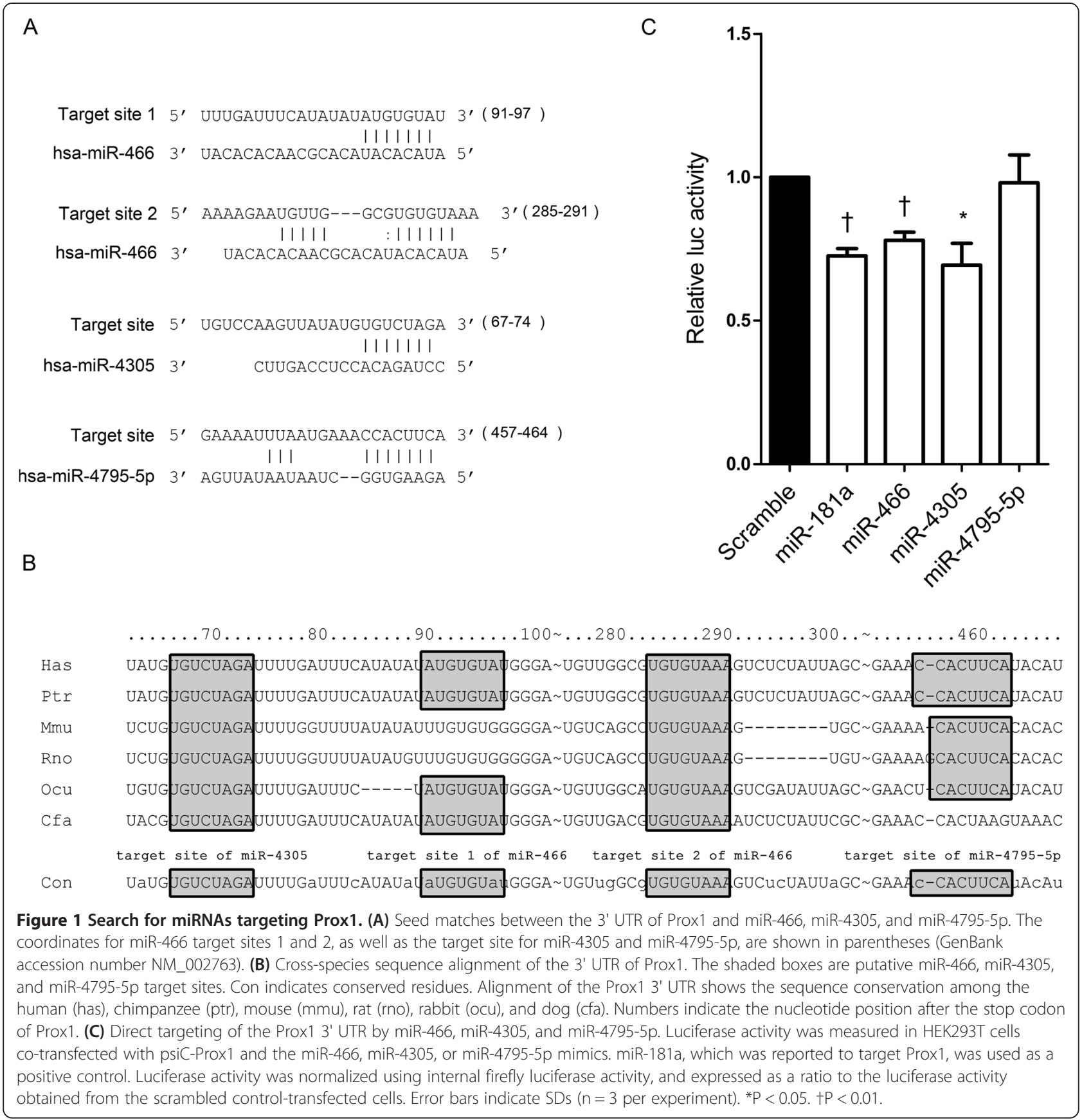

specific effects, we used 10-20 nM miRNA mimics throughout the experiments.

\section{Confirming target sites for miR-466 in the Prox1 3' UTRs}

The 3' UTR of Prox1 contains two putative binding sites for miR-466 (Figure 1A). To test whether both were directly targeted by miR-466, point mutations were introduced to psiC-Prox1 to produce psiC-Prox1-m1, psiCProx1-m2, and psiC-Prox1-m1m2 (Figure 4A and B). Each of these vectors was co-transfected with the miR466 mimic into HEK293T cells, and the luciferase assay was conducted. Luciferase activity was partially reduced in the cells transfected with the miR-466 mimic together with either psiC-Prox1 or psiC-Prox1-m1 (Figure 4C). However, luciferase activity was unaffected in the cells transfected with the miR-466 mimic together with psiCProx1-m2 (Figure 4C). As expected from the fact that both of the putative seed match sites were eliminated, luciferase activity was not affected when psiC-Prox1$\mathrm{m} 1 \mathrm{~m} 2$ was co-transfected with miR-466 (Figure 4C). Luciferase activity was not affected in the cells cotransfected with wild-type or mutant Prox1 3' UTR 
reporter vectors and miR-466 $\mathrm{m}$ (mutant form of miR466 ) or the scrambled control (Figure 4C). These results showed that target site 2 , but not the target site 1 , on the 3' UTR of the Prox1 was targeted by miR-466.

\section{Effect of miRNAs on tube formation in HDLEC}

To test whether miR-466 has an anti-lymphangiogenesis effect, an in vitro tube formation assay was conducted. Forty eight hours after transfection with miR-466 mimic, HDLEC were cultured on a Matrigel-coated 96 well plate for 4-6 $\mathrm{h}$ and the extent of tube formation was assessed. Formation of a rich network of tubular structures was observed in HDLEC transfected with the scrambled miRNA. miR-466 mimic significantly impaired the tube-forming activity of HDLEC as compared to the scrambled control (Figure 5A). Tube formation was then quantified by measuring the number of tubes formed using image manipulation software (ImageJ). The miR-181a mimic exerted the highest level of inhibitory effects on tube formation (approximately $84 \%$ ) followed by the miR-466 mimic (approximately 57\%; Figure 5B) when compared to the scrambled control. We also tested whether the inhibition of miR-466 enhanced tube formation in HDLEC and whether Prox1 siRNA counteracted the effect of a miR-466 inhibitor. As expected, Prox1 siRNA transfection significantly decreased Prox 1 expression in HDLEC as compared to transfection with the scrambled control (Figure 5C). HDLEC were then transfected with a miR-466 inhibitor alone or together with Prox1 siRNA. HDLEC transfected with the miR-466 inhibitor alone showed significantly increased tube formation (Figure 5D and E). However, cotransfected Prox1 siRNA counteracted the effect of the miR-466 inhibitor (Figure 5D and E).

Effect of miRNAs on angiogenesis and lymphangiogenesis in the corneal alkali burn animal model

The effects of miRNAs on corneal opacity, angiogenesis, and lymphangiogenesis were examined in an experimental animal model. Immediately after inducing an alkali burn, each miRNA mimic was subconjunctivally injected once, and the effect of each miRNA was evaluated two weeks later. miR-181a was used as a positive control, while the scrambled miRNA was used as a negative control. In the scrambled control-injected animals, the injured central corneal stroma appeared opaque with a distinct edematous margin (Figure 6A). In contrast, miR-466- or miR-181ainjected animals showed reduced opacity (Figure 6B and 6C). Under direct microscopic observation, blood vascular infiltration was about $46 \%$ of the scrambled control group level in the miR-181a-treated group. When the animals were injected with miR-466, blood vascular infiltration was $51 \%$ of the scrambled control group level (Figure 6J). Infiltration of blood vessel into the cornea was examined by staining with phalloidin to detect F-actin (Figure 6D-F), and positive cells were quantified using the ImageJ software. The cornea section from the scrambled miRNAinjected eyes showed a thickened cornea and strong scattered F-actin staining. In the miR-181a-treated group, the cornea showed almost half the thickness and F-actin positive staining was about $38 \%$ compared to the scrambled control group (Figure 6K). When the animals were injected with miR-466, corneal thickness was between those observed for the scrambled control and the miR-181ainjected animals, while F-actin positive staining was $56 \%$ of the scrambled control group level (Figure 6K). To analyze the effects of miRNAs on lymphangiogenesis of the cornea, corneal sections from each animal group were analyzed by immunohistofluorescence staining with anti-LYVE-1 antibodies (Figure 6G-I). miR-466- and miR-181a-injected corneas showed significantly reduced levels of LYVE-1 staining ( $\sim 33 \%$ and $\sim 30 \%$, respectively) compared to the scrambled control-treated corneas (Figure 6L).

\section{Discussion}

The results of the current study showed that the expression of Prox1 was inhibited by miR-466 at both the mRNA and protein levels. The luciferase assay showed that miR-466 directly targeted the well conserved 7 mer1A site in the 3' UTR of Prox1 (target site 2), as the suppressive effect of the miR-466 mimic on luciferase activity was almost abolished when this site was mutated. The target site 2 , unlike the target site 1 , was well conserved among species. Previous reports showed that additional Watson-Crick pairing to four or five sequential nucleotides at nucleotides 12-17 enhanced miRNA targeting [24]. The target site 2 contains five contiguous sequences that can be used for an effective 3' pairing with the nucleotides $14 \sim 18$ of miR-466, while the target site 1 does not. Thus, the target site 2 seems to have better chance to be targeted with miR-466 than the target site 1 .

In the alkali burn corneal injury model, miR-466 decreased corneal opacity and inhibited both lymphangiogenesis and angiogenesis, possibly attributable to decreased Prox1 expression. According to the results of a recent study [25], growth and migration of vascular endothelial cells were decreased by the addition of culture supernatant derived from Prox1 siRNA-treated oral squamous cell carcinoma cell cultures. In that experiment, down-regulation of VEGF-C was observed following silencing of the Prox1 gene [25]. These results suggested that Prox1 acted as a regulator of angiogenesis and lymphangiogenesis in oral squamous cell carcinoma [25]. Prox1 is known to control the expression of angiopoietin-2, which promotes angiogenesis in vascular endothelial cells [26]. VEGF-C and angiopoietin-2 are also important modulators of angiogenesis [27,28]. Therefore, suppressed Prox1 expression by miR-466 may have inhibited the expression of angiogenic modulators such as VEGF-C and angiopoietin-2, resulting 


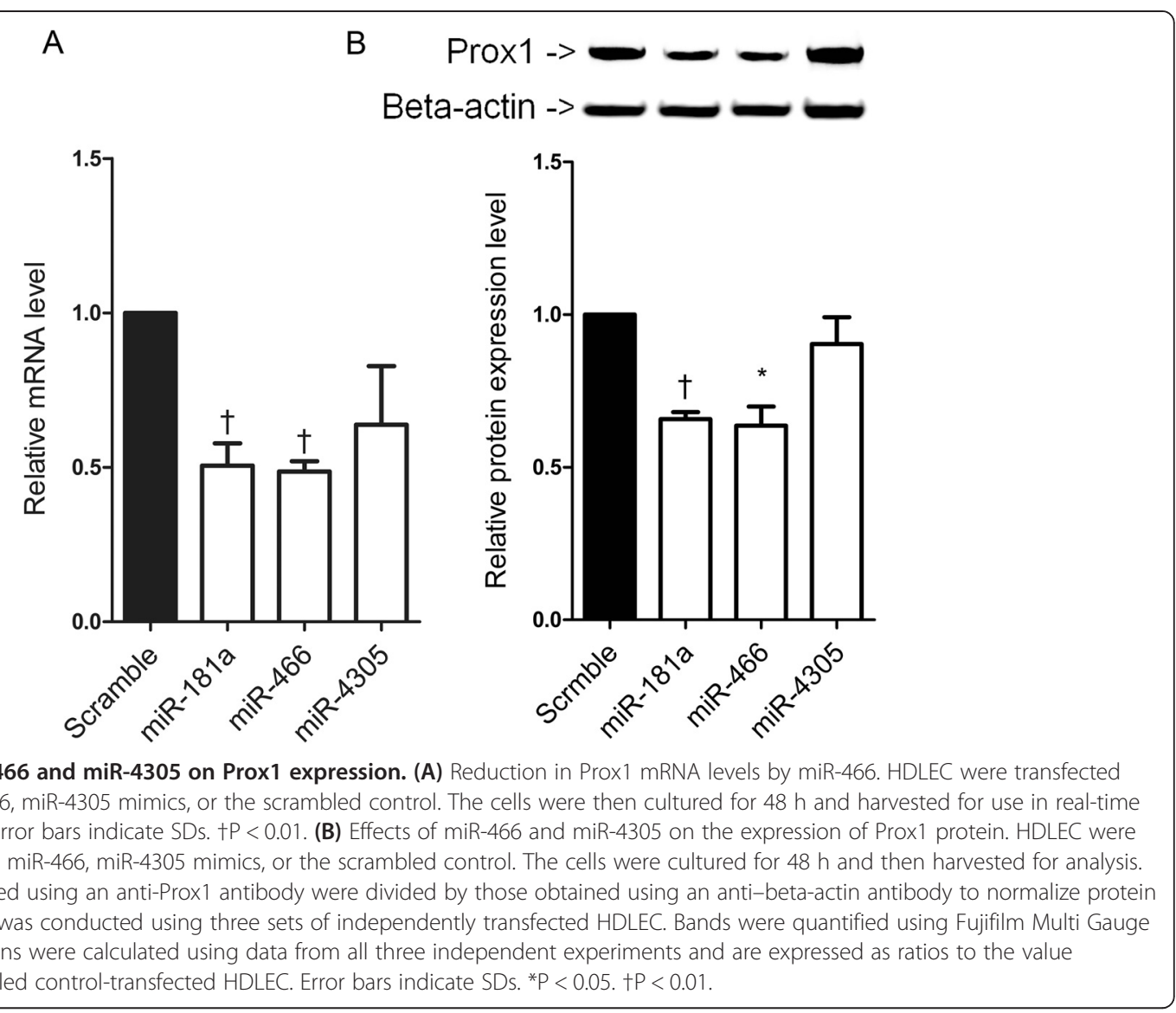

in reduced angiogenesis in the alkali burn corneal injury animal model. However, as miRNAs usually have multiple targets, our observations may be attributable to angiogenic modulators other than Prox 1 targeted by miR-466. This notion is supported by previous findings demonstrating that miR-466 induced apoptosis by targeting a few anti-

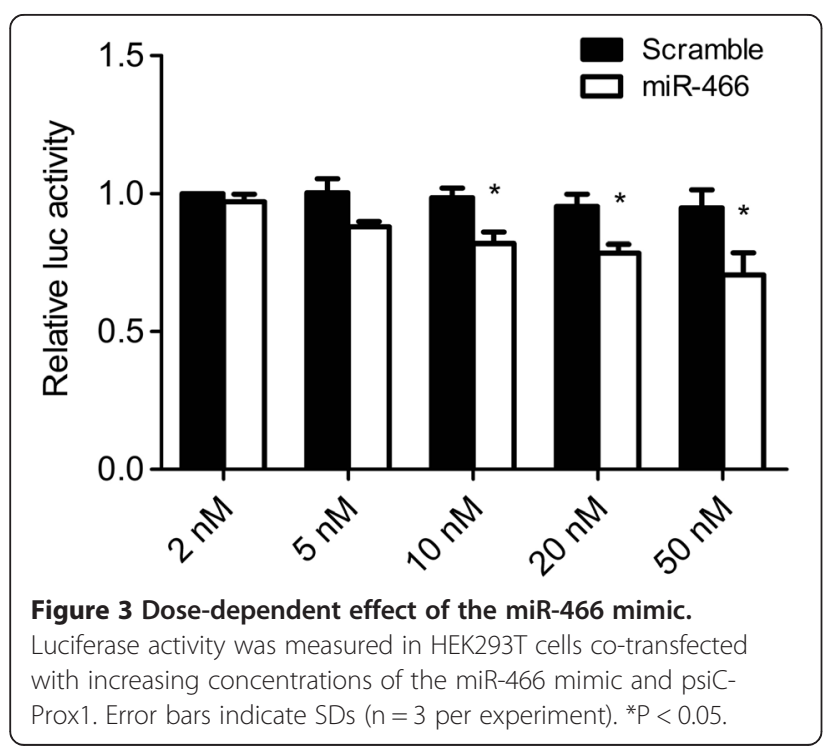

apoptotic genes [29], and that this induced apoptosis resulted in the inhibition of angiogenesis [30,31].

mmu-miR-466, which can be induced by apoptosis [29] and metabolic oxidative stress [32], was shown to enhance viral replication by inhibition of INF- $\alpha$ [33]. In addition, over-expression of mmu-miR-466 inhibited Nfat5 expression and was associated with renal dysfunction [34]. However, the functions of hsa-miR-466 in human cells have yet to be fully elucidated. The results of the current study demonstrated that hsa-miR-466 inhibited Prox1 expression and suppressed tube formation in human primary lymphatic endothelial cells. Furthermore, miR-466 reduced angiogenesis and lymphangiogenesis, resulting in clearer corneas than those observed in the scrambled control-treated mice in an animal cornea injury model.

The inhibitory effects of miR-466 on Prox1 expression, tube formation, and lymphatic vessel formation were comparable to those of miR-181. However, the inhibitory effect of miR-466 on blood vessel formation in the in vivo corneal injury model was slightly weaker than that of miR-181a. Findings of discrepant efficiencies attributed to miRNAs measured using different methods are not rare $[35,36]$ and may be due to different experimental settings, or may reflect experimental errors. Another 


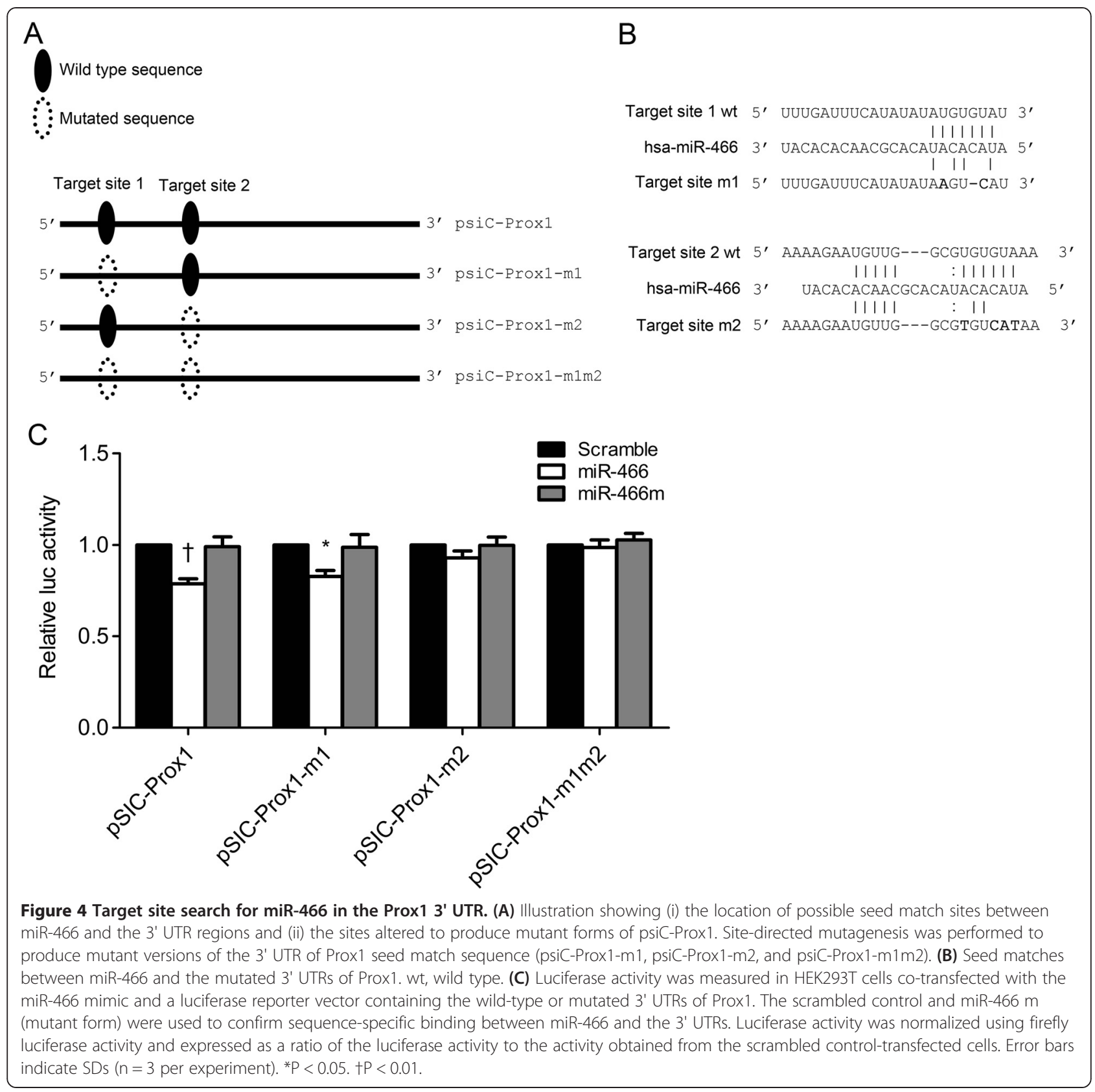

possibility is that miR-181a may also target angiogenesisrelated genes other than Prox1 more effectively than miR-466.

miR-4305 also significantly reduced the luciferase activity of the Prox1 3' UTR reporter vector as compared to the scrambled control in this study. However, Prox1 expression was not changed following miR-4305 mimic transfection of HDLEC compared to the scrambled control transfection. It is not clear why the miR-4305 mimic showed discrepant results in the luciferase assay, and in qRT-PCR and western blot experiments. These discrepant findings may be due to the use of only the 3' UTR of
Prox1, as opposed to the whole Prox 1 mRNA, in the luciferase assay. The seed match sequence on the 3' UTR of Prox1 in the luciferase reporter construct may have been available for miR-4305 binding, while the confirmation of that sequence in the Prox1 mRNA may have not allowed for miR-4305 binding. In addition, HEK293T cells were used for the luciferase assay, while HDLEC were used for other experiments.

Bevacizumab is a recombinant humanized immunoglobulin G1 monoclonal antibody against all isoforms of VEGF-A [37]. Bevacizumab binds VEGF-A and prevents the interaction of VEGF-A to its receptor on the surface 


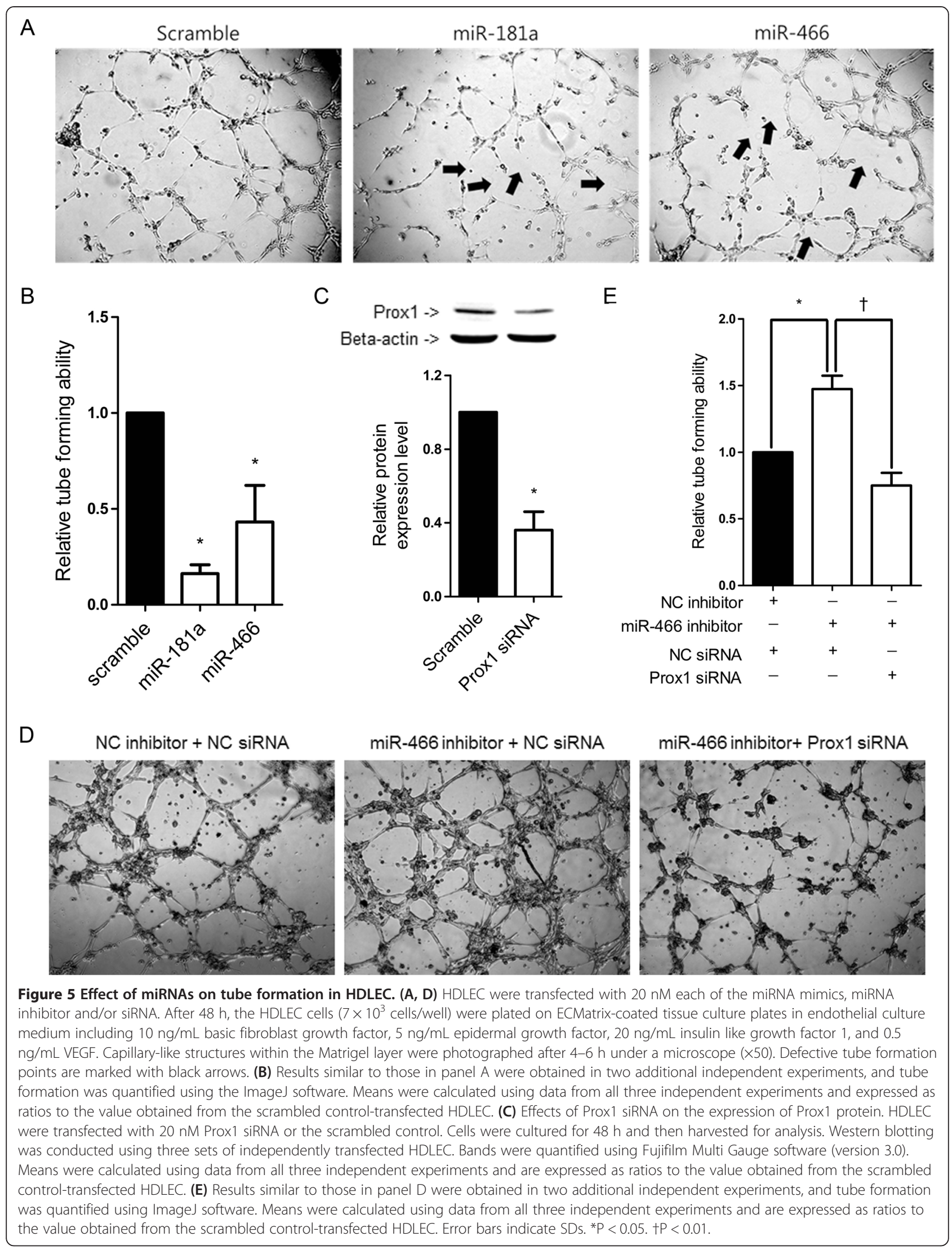



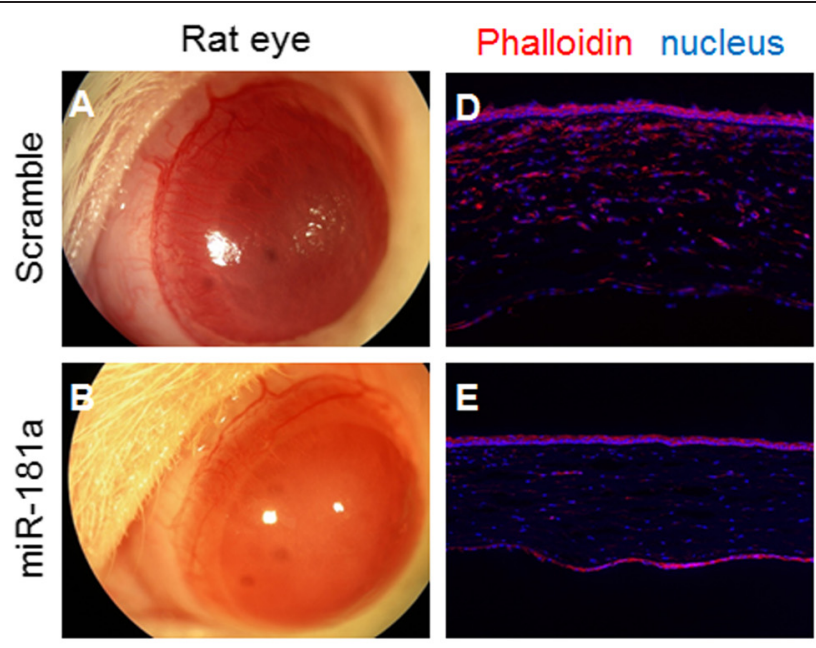

\section{LYVE-1 nucleus}
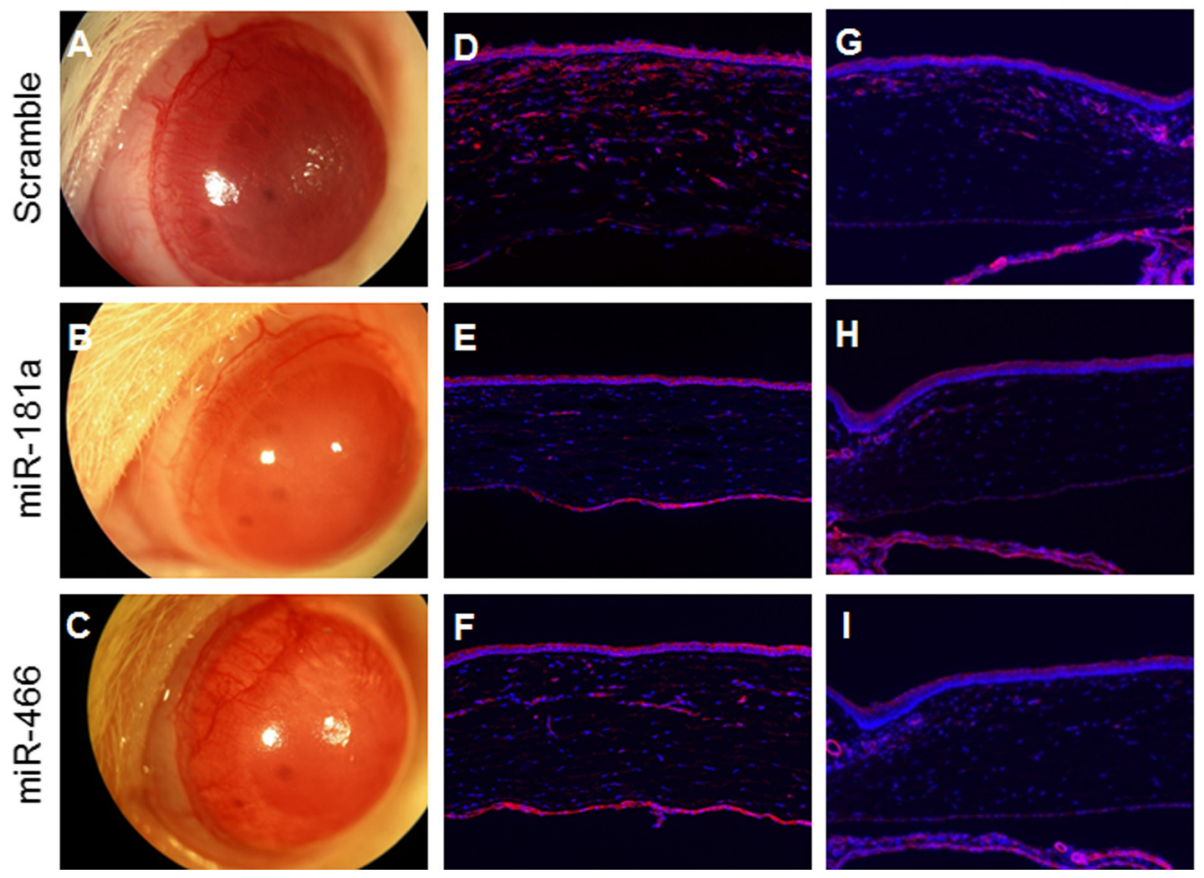

$\mathrm{J}$

K

$\mathrm{L}$
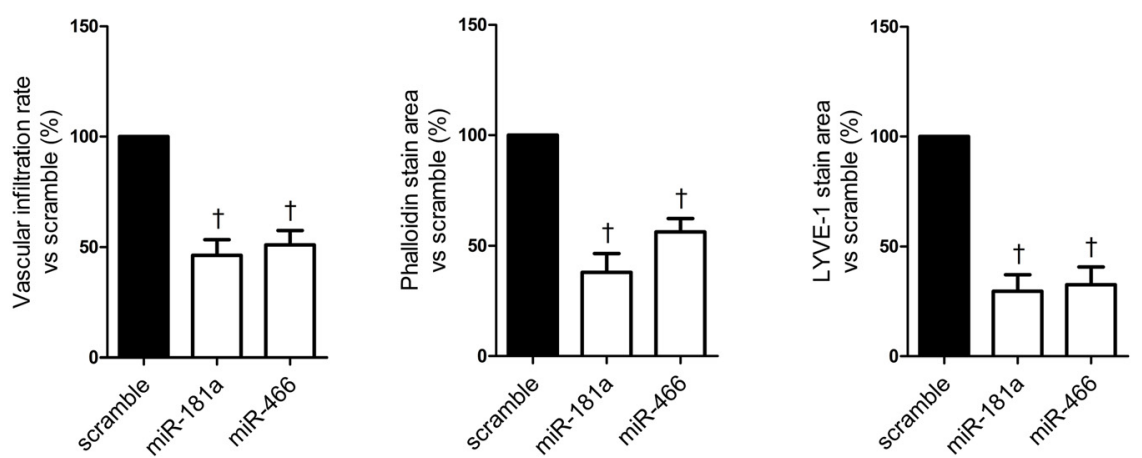

Figure 6 Inhibition of corneal neovascularization by miRNAs in a corneal injury animal model. Alkali burn-induced corneas of SpragueDawley rats were treated once with each miRNA mimic by subconjunctival injection on the day of injury. (A-C) Photographic images of corneas treated with each miRNA mimic. Immunofluorescent stain of corneal sections to detect vessel formation (D-F) or lymphangiogenesis (G-I). Red stains F-actin (D-F) or LYVE-1 (G-I), which is a marker of vessels and lymphatic vessels, and blue shows hoechst staining (D-I). Photographs of corneas were taken two weeks after the treatment. (J) Quantification of new blood vessel formation, F-actin-stained area (K), and LYVE-1-stained area (L). Relative rate was quantified using ImageJ software and normalized to the value obtained from the scrambled control-injected rats. Error bars indicate SDs. $+P<0.01$.

of endothelial cells, leading to inhibition of endothelial cell proliferation and new blood vessel formation [38]. Since the FDA approved bevacizumab for the treatment of metastatic colorectal cancer in combination with 5-fluorouracil, bevacizumab has been used for various malignancies [38,39]. Although bevacizumab-based treatment on eye diseases has not been approved by the FDA, intravitreal bevacizumab injection led to improvement of visual acuity and regression of retinal neovascularization in proliferative diabetic retinopathy patients [40]. Likewise, intraocular pressure and angiogenesis decreased following the administration of bevacizumab eye drops in neovascular glaucoma patients [41]. Furthermore, subconjunctival administration of bevacizumab after corneal transplantation decreased the number and caliber of vessels, however the effects were transient [42]. Bevacizumab inhibits only VEGF-A-induced lymphangiogenesis and has no effects on the lymphangiogenesis caused by VEGF-C or VEGF-D [43]. To treat lymphangiogenesis-related disease more effectively, it is necessary to develop drugs that directly target the process of lymphangiogenesis. 
The results of the current study demonstrated that miR-466 inhibited Prox1, which is known to be activated by various growth factors including VEGF-A, $-\mathrm{C}$, and $-\mathrm{D}$ $[11,44]$. Thus, miR-466 is expected to have broader and greater inhibitory effects on lymphangiogenesis-related diseases than bevacizumab.

\section{Conclusions}

Our results on human lymphatic endothelial cell and alkali burn corneal injury model demonstrated that miR466 directly targeted the 3 ' UTR of Prox 1 and suppressed the expression of Prox1, resulting in inhibition of lymphangiogenesis. Therefore, miR-466 may be useful for investigating the mechanisms of lymphangiogenesis and for developing treatments for lymphangiogenic eye diseases.

\section{Additional file}

Additional file 1: TableS1. Predicted miRNAs on target Prox1 gene (TargetScan 6.2, http://www.targetscan.org/).

\section{Abbreviations}

VEGF: Vascular endothelial growth factor; VEGFR: VEGF receptor; Prox1: Prospero homeobox 1; miRNA: MicroRNA; 3' UTR: 3' untranslated regions; HDLEC: Human primary lymphatic endothelial cell; GAPDH: Glyceraldehyde phosphate dehydrogenase; LYVE: Lymphatic vessel endothelial hyaluronan receptor; qRT-PCR: Quantitative reverse transcriptionpolymerase chain reaction.

\section{Competing interests}

The authors declare that they have no competing interests.

\section{Authors' contributions}

MS and SKL designed overall experiments and wrote the manuscript. MS carried out the in vitro experiments. JC, CRR and CJ discussed the experimental design and participated in manuscript writing. JC, CRR carried out the in vivo experiments. All authors read and approved the final manuscript.

\section{Acknowledgements}

This study was supported by a grant of the Korean Health Technology R\&D Project, Ministry for Health \& Welfare, Republic of Korea. (HI09C1555), and by the GRRC program of Gyeonggi province [2014-B05, Development of bio-medical lead compounds using RNAi].

\section{Author details}

${ }^{1}$ Department of Medical Lifescience, College of Medicine, The Catholic University of Korea, Seoul, Korea. ${ }^{2}$ Catholic Institute for Visual Science, College of Medicine, The Catholic University of Korea, Seoul, Korea. ${ }^{3}$ Department of Ophthalmology and Visual Science, Daejeon St. Mary's Hospital, Daejeon, Korea. ${ }^{4}$ Department of Ophthalmology and Visual Science, Seoul St. Mary's Hospital, Seoul, Korea.

Received: 20 August 2014 Accepted: 3 December 2014

Published online: 02 January 2015

\section{References}

1. Panda A, Vanathi M, Kumar A, Dash Y, Priya S: Corneal graft rejection. Surv Ophthalmol 2007, 52:375-396.

2. Dawson DW, Volpert OV, Gillis P, Crawford SE, Xu H, Benedict W, Bouck NP: Pigment epithelium-derived factor: a potent inhibitor of angiogenesis. Science 1999, 285:245-248.

3. Zhang P, Wu D, Ge J, Zhu Z, Feng G, Yue T, Lin J, Zheng H: Experimental inhibition of corneal neovascularization by endostatin gene transfection in vivo. Chin Med J (Engl) 2003, 116:1869-1874.
4. Ling $S$, Lin H, Xiang D, Feng G, Zhang X: Clinical and experimental research of corneal lymphangiogenesis after keratoplasty. Ophthalmologica 2008, 222:308-316.

5. Cursiefen C, Chen L, Dana MR, Streilein JW: Corneal lymphangiogenesis: evidence, mechanisms, and implications for corneal transplant immunology. Cornea 2003, 22:273-281.

6. Liu Y, Hamrah P, Zhang Q, Taylor AW, Dana MR: Draining lymph nodes of corneal transplant hosts exhibit evidence for donor major histocompatibility complex (MHC) class II-positive dendritic cells derived from MHC class II-negative grafts. J Exp Med 2002, 195:259-268.

7. Otrock ZK, Mahfouz RA, Makarem JA, Shamseddine Al: Understanding the biology of angiogenesis: review of the most important molecular mechanisms. Blood Cells Mol Dis 2007, 39:212-220.

8. Matsumoto T, Claesson-Welsh L: VEGF receptor signal transduction. Sci STKE 2001, 2001:re21.

9. Joukov V, Pajusola K, Kaipainen A, Chilov D, Lahtinen I, Kukk E, Saksela O, Kalkkinen N, Alitalo K: A novel vascular endothelial growth factor, VEGF-C, is a ligand for the Flt4 (VEGFR-3) and KDR (VEGFR-2) receptor tyrosine kinases. Embo j 1996, 15:1751.

10. Achen MG, Jeltsch M, Kukk E, Makinen T, Vitali A, Wilks AF, Alitalo K, Stacker SA: Vascular endothelial growth factor D (VEGF-D) is a ligand for the tyrosine kinases VEGF receptor 2 (Flk1) and VEGF receptor 3 (Flt4). Proc Natl Acad Sci U S A 1998, 95:548-553.

11. Tammela T, Alitalo K: Lymphangiogenesis: Molecular mechanisms and future promise. Cell 2010, 140:460-476.

12. Oliver G, Sosa-Pineda B, Geisendorf S, Spana EP, Doe CQ, Gruss P: Prox 1, a prospero-related homeobox gene expressed during mouse development. Mech Dev 1993, 44:3-16.

13. Wigle JT, Harvey N, Detmar M, Lagutina I, Grosveld G, Gunn MD, Jackson DG, Oliver G: An essential role for Prox 1 in the induction of the lymphatic endothelial cell phenotype. Embo j 2002, 21:1505-1513.

14. Hong YK, Harvey N, Noh YH, Schacht V, Hirakawa S, Detmar M, Oliver G: Prox 1 is a master control gene in the program specifying lymphatic endothelial cell fate. Dev Dyn 2002, 225:351-357.

15. Hong YK, Detmar M: Prox1, master regulator of the lymphatic vasculature phenotype. Cell Tissue Res 2003, 314:85-92.

16. Dietrich T, Bock F, Yuen D, Hos D, Bachmann BO, Zahn G, Wiegand S, Chen $L$, Cursiefen C: Cutting edge: lymphatic vessels, not blood vessels, primarily mediate immune rejections after transplantation. J Immunol 2010, 184:535-539.

17. Flister MJ, Wilber A, Hall KL, Iwata C, Miyazono K, Nisato RE, Pepper MS, Zawieja DC, Ran S: Inflammation induces lymphangiogenesis through up-regulation of VEGFR-3 mediated by NF-kappaB and Prox1. Blood 2010, 115:418-429.

18. Petrova TV, Makinen T, Makela TP, Saarela J, Virtanen I, Ferrell RE, Finegold DN, Kerjaschki D, Yla-Herttuala S, Alitalo K: Lymphatic endothelial reprogramming of vascular endothelial cells by the Prox-1 homeobox transcription factor. Embo j 2002, 21:4593-4599.

19. Harvey NL, Srinivasan RS, Dillard ME, Johnson NC, Witte MH, Boyd K, Sleeman MW, Oliver G: Lymphatic vascular defects promoted by Prox1 haploinsufficiency cause adult-onset obesity. Nat Genet 2005, 37:1072-1081.

20. Perron MP, Provost $P$ : Protein interactions and complexes in human microRNA biogenesis and function. Front Biosci 2008, 13:2537-2547.

21. Kazenwadel J, Michael MZ, Harvey NL: Prox1 expression is negatively regulated by miR-181 in endothelial cells. Blood 2010, 116:2395-2401.

22. Pedrioli DM, Karpanen T, Dabouras V, Jurisic G, van de Hoek G, Shin JW, Marino D, Kalin RE, Leidel S, Cinelli P, Schulte-Merker S, Brandli AW, Detmar M: miR-31 functions as a negative regulator of lymphatic vascular lineage-specific differentiation in vitro and vascular development in vivo. Mol Cell Biol 2010, 30:3620-3634.

23. Arnaoutova I, George J, Kleinman HK, Benton G: The endothelial cell tube formation assay on basement membrane turns 20: state of the science and the art. Angiogenesis 2009, 12:267-274.

24. Grimson A, Farh KK, Johnston WK, Garrett-Engele P, Lim LP, Bartel DP: MicroRNA targeting specificity in mammals: determinants beyond seed pairing. Mol Cell 2007, 27:91-105.

25. Sasahira T, Ueda N, Yamamoto K, Kurihara M, Matsushima S, Bhawal UK, Kirita T, Kuniyasu H: Prox1 and FOXC2 act as regulators of lymphangiogenesis and angiogenesis in oral squamous cell carcinoma. PLoS One 2014, 9:e92534. 
26. Harada K, Yamazaki T, Iwata C, Yoshimatsu Y, Sase H, Mishima K, Morishita Y, Hirashima M, Oike Y, Suda T, Miura N, Watabe T, Miyazono K: Identification of targets of Prox1 during in vitro vascular differentiation from embryonic stem cells: functional roles of HoxD8 in lymphangiogenesis. J Cell Sci 2009, 122:3923-3930.

27. Augustin $\mathrm{HG}$, Koh GY, Thurston G, Alitalo K: Control of vascular morphogenesis and homeostasis through the angiopoietin-Tie system. Nat Rev Mol Cell Biol 2009, 10:165-177.

28. Cao Y, Linden P, Farnebo J, Cao R, Eriksson A, Kumar V, Qi JH, ClaessonWelsh L, Alitalo K: Vascular endothelial growth factor $C$ induces angiogenesis in vivo. Proc Natl Acad Sci U S A 1998, 95:14389-14394.

29. Druz A, Chu C, Majors B, Santuary R, Betenbaugh M, Shiloach J: A novel microRNA mmu-miR-466 $\mathrm{h}$ affects apoptosis regulation in mammalian cells. Biotechnol Bioeng 2011, 108:1651-1661.

30. Dimmeler S, Zeiher AM: Endothelial cell apoptosis in angiogenesis and vessel regression. Circ Res 2000, 87:434-439.

31. Folkman J: Angiogenesis and apoptosis. Semin Cancer Biol 2003, 13:159-167.

32. Druz A, Betenbaugh M, Shiloach J: Glucose depletion activates mmu-miR-466 h-5p expression through oxidative stress and inhibition of histone deacetylation. Nucleic Acids Res 2012, 40:7291-7302.

33. Li Y, Fan X, He X, Sun H, Zou Z, Yuan H, Xu H, Wang C, Shi X: MicroRNA$466 I$ inhibits antiviral innate immune response by targeting interferon-alpha. Cell Mol Immunol 2012, 9:497-502.

34. Luo Y, Liu Y, Liu M, Wei J, Zhang Y, Hou J, Huang W, Wang T, Li X, He Y, Ding F, Yuan L, Cai J, Zheng F, Yang JY: Sfmbt2 10th intron-hosted miR-466(a/e)-3p are important epigenetic regulators of Nfat5 signaling, osmoregulation and urine concentration in mice. Biochim Biophys Acta 1839, 2014:97-106.

35. Yan X, Chen X, Liang H, Deng T, Chen W, Zhang S, Liu M, Gao X, Liu Y, Zhao C, Wang X, Wang N, Li J, Liu R, Zen K, Zhang CY, Liu B, Ba Y: miR-143 and miR-145 synergistically regulate ERBB3 to suppress cell proliferation and invasion in breast cancer. Mol Cancer 2014, 13:220.

36. Renjie W, Haiqian L: MiR-132, miR-15a and miR-16 synergistically inhibit pituitary tumor cell proliferation, invasion and migration by targeting Sox5. Cancer Lett 2015, 356:568-578.

37. Ferrara N, Hillan KJ, Gerber HP, Novotny W: Discovery and development of bevacizumab, an anti-VEGF antibody for treating cancer. Nat Rev Drug Discov 2004, 3:391-400.

38. Syrigos KN, Karapanagiotou E, Boura P, Manegold C, Harrington K: Bevacizumab-induced hypertension: pathogenesis and management. BioDrugs 2011, 25:159-169.

39. Giantonio BJ, Catalano PJ, Meropol NJ, O'Dwyer PJ, Mitchell EP, Alberts SR, Schwartz MA, Benson AB 3rd: Bevacizumab in combination with oxaliplatin, fluorouracil, and leucovorin (FOLFOX4) for previously treated metastatic colorectal cancer: results from the Eastern Cooperative Oncology Group Study E3200. J Clin Oncol 2007, 25:1539-1544.

40. Spaide RF, Fisher YL: Intravitreal bevacizumab (Avastin) treatment of proliferative diabetic retinopathy complicated by vitreous hemorrhage. Retina 2006, 26:275-278.

41. Waisbourd M, Shemesh G, Kurtz S, Rachmiel R, Moisseiev E, Zayit-Soudri S, Loewenstein A, Barequet I: Topical bevacizumab for neovascular glaucoma: a pilot study. Pharmacology 2014, 93:108-112.

42. Awadein A: Subconjunctival bevacizumab for vascularized rejected corneal grafts. J Cataract Refract Surg 2007, 33:1991-1993.

43. Bock F, Onderka J, Dietrich T, Bachmann B, Kruse FE, Paschke M, Zahn G, Cursiefen C: Bevacizumab as a potent inhibitor of inflammatory corneal angiogenesis and lymphangiogenesis. Invest Ophthalmol Vis Sci 2007, 48:2545-2552.

44. Hirakawa S, Kodama S, Kunstfeld R, Kajiya K, Brown LF, Detmar M: VEGF-A induces tumor and sentinel lymph node lymphangiogenesis and promotes lymphatic metastasis. J Exp Med 2005, 201:1089-1099.

\section{Submit your next manuscript to BioMed Central and take full advantage of:}

- Convenient online submission

- Thorough peer review

- No space constraints or color figure charges

- Immediate publication on acceptance

- Inclusion in PubMed, CAS, Scopus and Google Scholar

- Research which is freely available for redistribution

Submit your manuscript at www.biomedcentral.com/submit 